\title{
La Educación Colombiana en la Década de los 80
}

\author{
Luis Hernando Rincón Bonilla \\ Universidad del Cauca, Popayán, Colombia
}

\section{Introducción}

El presente ensayo tiene como finalidad presentar una visión general de la evolución de la educación en Colombia en la década de 1980. Dada la vastedad del tema la reflexión se centrará en los siguientes aspectos: en primer lugar el papel que se asigna a la educación en los planes de desarrollo de los distintos gobiernos de la década, luego se analizan los programas y reformas educativas más importantes que se impulsan en el período los programas de formación y capacitación de docentes y finalmente algunas consideraciones sobre las perspectivas que se vislumbran para la educación colombiana con la constitución de 1991. Este documento recoge el resultado de algunos trabajos, investigaciones, documentos y mis reflexiones personales sobre el tema y, por consiguiente, más que un texto acabado es un material perfectible.

\section{La educación en los planes de desarrollo}

A pesar de Colombia haber iniciado formalmente un proceso de modernización desde mediados del siglo, el impacto sólo se ha comenzado a sentir de manera determinante en las dos últimas décadas en todas las instituciones de la sociedad civil.

En el campo de la educación, a pesar de que desde 1958 cuando se inició el período denominado el Frente Nacional, o Bipartidismo se intentó impulsar, con criterios de racionalidad y eficacia, el desarrollo del sector, lo cierto es que el sistema educativo ha permanecido en una crisis agobiante en donde ha imperado la improvisación y el espontaneismo que han dejado las múltiples propuestas de cambio en meros enunciados.

En la generalidad de los planes de desarrollo del país, en las dos últimas décadas, la educación fue centrada en la metáfora de la "formación de los recursos humanos". "La articulación entre educación y sociedad o la contribución de la educación al desarrollo, comienza a ser percibida básicamente a través del eje económico; el supuesto del cual partía esta concepción era que 
más años de estudio implicaban mayor productividad, mayores salarios, y mayor equidad en la distribución de los beneficios. A partir de es supuesto, la educación comienza a ser considerada un área donde es posible adoptar estrategias planificadoras, donde las decisiones en materia de inversiones educativas pueden ser efectuadas en los mismos términos que cualquier inversión económica" (Tudesco, 1990)

Sin embargo, a principios de los 80 esta concepción comienza evidenciar serios signos de crisis, debido a que el crecimiento de la economía del país, no alcanza los niveles esperados y por lo tanto su crecimiento muestra índices marcados de contracción, las necesidades de recursos humanos se cubren rápidamente y se observan altas tasas de desempleo y subempleo, subutilización de capacidades y de desajuste entre el nivel educativo y el trabajo desempeñado. Además, la expansión del sistema educativo que fue significativo en las década precedentes ( 60 y 70 especialmente) no fue acompañado por otros cambios sociales, que lograran superar las barreras estructurales de la sociedad colombiana; es decir, la educación deja de ser concebida como ascensor social y como salvavidas laboral.

A partir de 1975 cuando se promulga la ley de nacionalización de la educación, que busca estabilizar la participación de la nación en los ingresos provenientes del impuesto a las ventas destinadas a los gastos de educación y a procurar una mayor participación de las regiones y las localidades en el financiamiento de los programas educativos, se muestra claramente un acelerado descenso en la cobertura educativa, en un momento cuando un porcentaje muy importante de la población no había logrado acceso a ese servicio y cuando aproximadamente el 50\% de áreas rurales y urbano marginales, no lograba superar los dos o tres primeros grados de enseñanza escolar y cuando las tasas de analfabetismo absoluto o por desuso, eran todavía significativas.

En general, los gobiernos de la década, se comprometieron en sus estrategias con el mejoramiento cualitativo y de adecuación de los programas; mientras en la década de los setenta, especialmente en el gobierno de Julio César Turbay Ayala (1978-1982) se formula el P.I.N. (Plan de Integración Nacional), que en materia educativa es la continuidad del plan anterior "Para cerrar la Brecha” de la administración López Michelsen (1974-1978); que en esencia buscan transferir responsabilidad y gastos de financiamiento de la educación a los Departamentos y Municipios. En la educación superior, se considera como una estrategia fundamental el autofinanciamiento a través del alza de matrícula.

Desde el punto de vista administrativo, se refuerza el mecanismo de la regionalización, mediante la ampliación del "mapa educativo" que permitirá 
organizar los establecimientos y programas desde el preescolar hasta la media vocacional en distritos y núcleos. En la Educación Superior se concreta la reforma de la Educación Superior, mediante el Decreto Ley 80 de 1980, que sería objeto de análisis posterior.

La administración de Belisario Betancourt (1982-1986) en su plan de desarrollo denominado "Cambio con Equidad" presenta para el sector educativo, las siguientes políticas: Modernización, descentralización y planificación participante, que supone la adopción de nuevos métodos e instrumentos educativos, la adopción de innovaciones y adecuación de la estructura administrativa.

Mejoramiento cualitativo, que busca la puesta al día de la currícula, su organización, según las peculiaridades regionales y la adopción de los supuestos y la metodología de la llamada escuela activa.

Se propone una campaña masiva de alfabetización denominada "Camina”, en la Educación Superior, la estrategia de impulso se focaliza en el programa de Universidad Abierta y a Distancia que llevó a mirarla como una panacea para solucionar los endémicos problemas que afectan a las universidades estatales.

Se impulsó el programa Escuela Nueva para las zonas rurales y se continuó con la nuclearización educativa.

En el gobierno de Virgilio Barco (1986-1990) se continúa con la estrategia de adecuar la educación al desarrollo del país, para ello se enfatiza en el mejoramiento de la calidad y la implementación masiva de la tecnología educativa. El programa induce la reorientación de los recursos educativos, priorizando la educación básica mediante la universalización de la educación de niños, jóvenes y adultos. Se promueven acciones de ampliación de la cobertura muy restringida, centrando la acción del gobierno en los programas de descentralización educativa que generó serios conflictos con el magisterio y otros sectores populares.

Los avances en la reorganización escolar del país que fue el objetivo eje del plan, fueron muy limitados en sus alcances en la cobertura, dinamización y separación de las barreras económicas y sociales.

En conclusión, a pesar de la profusión de planes y programas que para el sector educativo, se formularon en los distintos gobiernos de la década, la educación Colombiana no mostró en el período, signos de mejoramiento de la crisis que ha afectado especialmente en su calidad, financiación, organización y cobertura. 


\section{Panorama de la Educación Colombiana en el Siglo XX}

El estudio de la evolución de la educación Colombiana, ha sido tema de múltiples investigaciones a partir de la década del sesenta, desde diferentes perspectivas que han abordado la descripción del fenómeno educativo en nuestro país.

Muchos de los trabajos realizados se han circunscrito a aspectos cuantitativos, y a elementos descriptivos de la legislación educativa; además de la atención se ha centrado con preferencia en la segunda mitad del siglo, en la que se han presentado fenómenos demográficos complejos, ligados a un aumento de la escolaridad. Acompañados de reformas educativas, especialmente durante los gobiernos del Frente Nacional.

En lo referente a las primeras décadas del presente siglo, no son muy numerosas las investigaciones, exceptuando la que se viene realizando en el marco del proyecto "Historia de la Pedagogía en Colombia", el estudio más coherente que sobre la temática se viene adelantando en el país.

En general, los estudios coinciden en señalar que la educación Colombiana no ha desarrollado una concepción teórica fundamental en la investigación científica de la realidad del país, acorde con los requerimientos, las posibilidades y expectativas de nuestra población.

Ha predominado la influencia de escuelas y tendencias pedagógicas importadas de España, Italia, Francia, Alemania, Inglaterra y los Estados Unidos: "cualquier reseña de las tendencias educativas de nuestro país pone de frente el transplante mecánico y sistemático de modelos foráneos y una ausencia protuberante de interés por la investigación pedagógica en nuestro medio. (Herrera, 1986: 90)

En general desde la década de los sesenta, nuestro sistema educativo en sus afanes de modernización ha tratado de implementar estrategias de carácter tecnológico diseñados en otras sociedades para los sectores más pobres de la población ofreciéndoles lo mínimo que en educación se necesita; si bien se ha incrementado la cobertura la calidad ha desmejorado, lo que ha contribuido a que la escuela pública rural y urbana marginal se haya convertido en una institución que ha contribuido a fragmentar la sociedad colombiana, agobiada además por una serie de problemas financieros, administrativos y curriculares, todo ello acompañado de un acentuado crecimiento de la educación privada que ha incrementado su oferta a los sectores de clase media de la sociedad colombiana.

El estudio realizado por Aline Helg, revela que esta tendencia comenzó a consolidarse desde la primera mitad de este siglo, especialmente no la reforma 
educativa del Gobierno del Presidente López Pumarejo que pretendía "desarrollar y modernizar al país para volverlo competitivo con el Mercado Capitalista Mundial, sin tocar las bases de la Estructura Social.

De acuerdo a los planteamientos de la teoría de la reproducción, según la cual en las sociedades capitalistas existen dos canales de formación, uno dirigido a las clases dominantes, es decir la educación privada, y otro dirigido al resto de la población, la educación pública.

Estos cambios tienen significativas diferencias en el grado de adquisición de los conocimientos y el desarrollo de habilidades; así como las oportunidades para el desarrollo del individuo y la permanencia en la escuela. afirma:

$\mathrm{Al}$ respecto Rodrigo Parra Sandoval en su libro "La Escuela Inconclusa"

La acelerada expansión del sistema escolar Colombiano durante las últimas cuatro décadas ha significado una ampliación del servicio educativo para una buena parte de la población en el edad de estudiar. Pero, al mismo tiempo, la posibilidad de asistir a la escuela no se ha distribuido de manera uniforme para todos los colombianos y ese hecho marca una más intensa discriminación para los que no asisten o permanecen menos años en la escuela. (Parra, 1986: 11)

El obligatorio cumplimiento de los programas oficiales en todas las escuelas del país, desconociendo que la Sociedad Colombiana se caracteriza por marcadas diferencias económicas, sociales, culturales y políticas que condicionan a la escuela y que exigen de las variaciones en sus funciones de acuerdo al contexto en que se desenvuelve. En Colombia los contextos sociales identificados son los siguientes:

Urbano en el cual es necesario diferencias el contexto con industrialización que se divide a su vez en marginal y no marginal, y los contextos urbanos sin industrialización.

En el contexto rural coexisten varios modos de producción; economías campesinas, para auto sustento y en menor escala para consumo urbano: economía de agricultura industrial, que producen materias primas para la industria y economías de agricultura empresarial caracterizadas por la presencia de empresarios agrícolas medios.

Según el estudio citado de Rodrigo Parra "puede en efecto, pensarse que la escuela lleva a cabo tres funciones fundamentales en la Sociedad Colombiana: "Una función de enseñanza, una función que hace relación a la producción, a la formación de mano de obra; una tercera función fundamental de la escuela en su rol de integradora de los individuos en valores y concepto que vienen de la sociedad más amplia.(Parra, 1986: 24.) 
El contenido programático evidencia quizá más que cualquier otro aspecto del sistema educativo que la imposición de un modelo esencialmente urbano que ha contribuido a atender el velo.

Que ha impedido apreciar los rasgos diferenciados de la escuela rural, inmersa en un contexto substancialmente distinto del propio de la escuela urbana. (FES, 1978: 4)

Como lo indica Rodrigo Parra:

Los problemas de la calidad de la educación, que constituye la deficiencia más notable del sistema escolar actual se siente con mayor rigor en la educación rural. Esto es asi debido a que por una parte la escuela se ha definido como un elemento integrador de la nacionalidad a partir de su función urbanizadora, lo que crea una dificultad adicional para la adaptación del curriculo único a la cultura rural y por otra parte, a que la escuela es un elemento extraño a la visión del mundo que los niños campesinos han adquirido en una socialización primaria. (Parra, 1986: 4)

El currículo imperante en la Educación Colombiana en las últimas décadas ha sido esencialmente urbano, pero no rural, razón por la cual la escuela rural se ha convertido en un enclave externo a las comunidades, que ha contribuido a la enajenación del niño y el joven campesino respecto a su medio, valores y costumbres, ha estimulado en cierto grado la migración del campo a la ciudad.

La rigidez curricular ha contribuido por otra parte a ampliar el abismo entre el desarrollo de la ciencia, los cambios tecnológicos que han ocasionado un gran impacto en todas las esferas de la vida y la inercia y precariedad de los procesos curriculares de nuestro sistema educativo.

A partir de la década de los sesenta el currículo ha sentido el impacto del modelo de la tecnología educativa fundamentada en la escuela conductista, que a través del diseño curricular redujo la práctica educativa a "un conjunto de actividades dispuestas como medios para alcanzar una serie de logros preestablecidos", se separó la concepción y diseño de los programas de su ejecución, convirtiendo de esta manera al maestro en un simple ejecutor de paquetes tecnológicos diseñados, para alcanzar una serie de objetivos conductuales previstos, lo que impide orientar el proceso de aprendizaje de acuerdo a las situaciones de aprendizaje de cada contexto en particular. La tecnología se dirigió de manera casi exclusiva a la educación pública, la educación privada si bien, lo ha incorporado lo ha hecho subordinada a algunos principios y además ha incorporado innovaciones provenientes de otras tendencias pedagógicas, como la escuela activa y piagetiana, psicoanálisis, educación personalizada, etc. 


\section{La Renovación Curricular}

Podemos remontar la historia de la Renovación Curricular a la década del sesenta, en la cual se dieron en el mundo fenómenos sociales y científicos muy importantes, que tuvieron serias repercusiones en políticas educativas de los países de América Latina. Se comenzó a impulsar masivamente la tecnología educativa de corte conductista como una panacea contra una de las causas del subdesarrollo de la región, la ignorancia. Los gobiernos de turno fueron asesorados por distintas fundaciones y organismos internacionales, quienes coincidieron en recomendar, que la mejor estrategia para optimizar el rendimiento del sistema educativo era la tecnología educativa como eje central de las políticas educativas.

Durante la década de los sesenta, la preocupación cambia de énfasis, es decir, de la cobertura se pasa al problema del mejoramiento de los procesos educativos, discusión que aún continúa vigente, la de la calidad de la educación. A partir de 1976 se inicia el diseño de un programa para el mejoramiento cualitativo de la educación dentro de la línea tecnología, el cual después de ser sometido a experimentación en algunas regiones del país y con varias previsiones, en los cuales el Magisterio Colombiano no tuvo participación, es oficiliazada para todo el país por medio del decreto 1002 del 24 de abril de 1986.

La Renovación Curricular comprende un trípode de documentos constituidos por: Los fundamentos teóricos (libro azul) que contienen los marcos legales epistemológicos, filosóficos, sociológicos, psicológicos y pedagógicos; los marcos generales de las áreas (libro amarillo) que conforman el plan de estudios de los distintos niveles del sistema educativo, y los programa específicos de las diferentes áreas por cursos. Los distintos estudios sobre las relaciones entre los fundamentos teóricos, los marcos generales y los programas, reflejan que existe entre ellos contradicción, pues mientras la lógica de los fundamentos se centra en la adquisición y construcción del conocimiento por parte del aprendiz, los marcos y los programas la subordinan a la teoría behaviorista de la transmisión al enfatizar en la utilización del discurso del diseño instruccional, teoría en la que la relación pedagógica tiene poca autonomía para estimular la participación, la autorregulación y la adecuación de los contenidos en los distintos contextos.

\section{La Formación y Capacitación de Docentes}

En general, la estructura, organización y desarrollo de los programas de formación y capacitación de docentes ha estado orientada a equiparlos de 
herramientas de enseñanza, de manera uniforme y mecánica, para el desarrollo de un .currículo único, en todos los contextos sociales, sin discriminación ni diferenciación; la utilización de modelos conductistas, al programar opciones únicas de conocimiento, no sólo frustran y limitan las posibilidades de elaboración colectiva del conocimiento, sino que perpetúan y agravan la inadecuada correspondencia entre los métodos, los contenidos de los programas y las necesidades del país y su población.

Tanto la capacitación como la formación se han orientado fundamentalmente a preparar al docente como un recurso transmisor y que por lo tanto desarrolla su práctica en consonancia con ella, asi el maestro, inmerso en esta estructura, enajena la condición del trabajo intelectual que realiza y se transforma en un "conductor" de sus alumnos, identificándose con el saber instituido, convirtiéndose en administrador y regulador del conocimiento que transmite a sus alumnos. Este juego de roles; maestro que sabe, alumno que no sabe, no sólo implica la ruptura de la unidad del enseñar y el aprender sino también la interiorización de la creencia o la fe, en la base del aprendizaje. (Batallan, 1983)

Los egresados de las escuelas normales tienen una formación muy limita$\mathrm{da}$, los programas que se desarrollan son muy tradicionales y progresivamente se los ha asimilado a bachilleres con unos fundamentos muy deficientes para su desempeño especialmente en las comunidades indígenas y rurales.

Los licenciados formados en la facultades de Educación tiene énfasis en sus programas para la escuela secundaria aun los licenciados en Español y Literatura, no han estudiado ni menos realizado prácticas en la enseñanza inicial. En las zonas rurales y los lugares más apartados es muy común encontrar maestros con títulos con nivel primario, u otros tipos de formación.

Se observa un círculo vicioso, parece como si la enseñanza en los niveles iniciales fuera un saber tan elemental que no vale tenerlo en cuenta, de ahí que sea uno de los principales problemas de nuestro sistema educativo, íntimamente ligado al fracaso escolar y a la baja calidad de la educación; es muy común observar los siguientes hechos:

1. Los maestros que enseñan en los primeros cursos ( 1 y 2 ) no tienen preparación específica para esta labor.

2. Los grupos primeros y segundos son los más numerosos y los que tienen menos apoyos materiales; bibliotecas, ayudas educativas, papelería, juegos educativos, etc.

3. La exigencia de la eficiencia por parte de la sociedad y los padres de familia en particular, que exigen que sus niños lean antes de finalizar el primer grado, le impiden al maestro dedicar tiempo para la lectura comprensiva. Todo parece indicar que esta difícil tarea no es comprendida por los demás. 
4. Es muy difícil encontrar en los maestros de la escuela primaria buenos lectores o personas que redacten con facilidad. Las encuestas y estudios realizados en diferentes regiones del país sobre los hábitos e intereses de lectura de los maestros colombianos, indican que nuestros educadores leen muy poco, sus lecturas se reducen a los libros de texto y otros materiales de poco contenido científico para su trabajo. Un grupo muy significativo ni siquiera conocer la Revista "Educación y Cultura”, una publicación de un gran contenido pedagógico publicada por la Federación Colombiana de Educadores.

5. Como lo hemos mencionado anteriormente, esta situación es más dramática en las comunidades indígenas, rurales y urbanas marginales.

6. La pérdida del estatus de la profesión del maestro, es otro factor que ha contribuido para que el maestro docente no se preocupe por mejorar su formación, utiliza su profesión como un medio de subsistencia o como un trampolín para tener una entrada económica y luego realizar otros estudios.

Queremos aclarar que la responsabilidad de esta situación no recae exclusivamente en los maestros, en ella intervienen las políticas del Estado y la sociedad en general.

La capacitación que se brinda a los maestros no es la más adecuada, en general, los maestros la han tomado como un requisito para ascender en el escalafón nacional más que como un medio de profesionalización y cualificación personal, de ahí que sea considerada como la "feria de los créditos"; además los recursos disponibles son muy limitados y generalmente los cursos se ofrecen en las zonas urbanas, descuidándose a los maestros rurales, quiénes tiene mayor necesidad de capacitación; por otra parte, los programas de capacitación son de bajo nivel, muy teóricos y no tienen secuencia ni continuidad.

A pesar de estarse implementando en el país la Renovación Curricular, los programas de capacitación de los docentes han sido muy deficientes. El proyecto de su implementación ha carecido de la decisión política, menos de lógica y coherencia, en realidad su efecto sobre la calidad de la enseñanza de la lengua materna no ha sido significativo, sigue predominando el modelo tradicional.

No se puede negar el interés de muchos educadores que, organizados y estimulados por el Movimiento Pedagógico, vienen desarrollando experiencias innovativas en la enseñanza de la lengua escrita, la gran cantidad de trabajo presentados al Congreso Pedagógico realizado en 1987 así lo demuestra; sin embargo, su impacto sigue siendo muy reducido, se requiere del apoyo 
decidido a estas experiencias tanto material como moralmente para que ellos se puedan generalizar en las de los distintos centros de educación del país.

La capacitación de los maestros en servicio necesita ser repensada. Los cursos de capacitación, donde los maestros reciben instrucciones para ser aplicados en el salón de clase no contribuyen a transformar su trabajo. Cuando se trata de contenidos que buscan generar rupturas con prácticas fuertemente arraigadas, es preciso poner primero en crisis los esquemas asimiladores de los maestros, para dar ocasión a que se reelaboren nuevos esquemas de ese tipo. Este proceso lleva tiempo, se debe respetar si se quiera que los maestros se apropien de las propuestas innovadoras. Se requiere también que la capacitación propicie una reflexión muy seria y profunda sobre la práctica cotidiana del maestro para que se tome conciencia de las contradicciones entre las intenciones y lo que realmente ocurre en el salón de clase. Creemos que esta es una tarea urgente en todas las áreas escolares, pero que es más apremiante en la alfabetización, ya que los niños que no logran franquear el umbral de la alfabetización inicial, tampoco lo podrán lograr en otras áreas programáticas. Es igualmente necesario que los maestros hagan un análisis de su comportamiento con respecto a la lengua escrita como lectores y usuarios de ella, sólo así será posible que se involucren como agentes activos en los procesos de capacitación.

En cuanto a la formación de maestros, se requiere transformar las orientaciones curriculares tanto en las Escuelas Normales como en las Facultades de Educación y demás centros de formación de docentes, de modo que el currículo contemple los contenidos socio-culturales, lingüístico s, psicológicos, pedagógicos y epistemológicos en una relación teoría práctica. Estos centros están muy lejos de ser laboratorios de experimentación pedagógica, están muy alejados de la práctica real y de muchos avances científicos que nutren la reflexión pedagógica.

Sólo un maestro formado con constructor del estado, de la ciencia y de la cultura puede ser un transformador social, sólo un maestro formado rigurosamente en las disciplinas cientificas, en la práctica pedagógica, en el conocimiento de nuestra historia y nuestra lengua, en el gusto por el arte y por la vida puede formar niños sanos, seguros de sr mismos conscientes de su responsabilidad histórica. (Caijao, 1987: 7)

\section{La Educación Superior en la Década}

Por medio del Decreto Ley NO. 080 del 22 de Enero de 1980, el Gobierno de Turbay establece la reforma de la Educación post- secundaria; reforma que se impone al ceder al Congreso su función legislativa al poder ejecutivo 
y sin un debate concienzudo tanto en la comunidad universitaria como en la opinión pública.

La reforma tuvo como objetivo básico la readecuación de la Universidad a las exigencias del modelo económico imperante en el país, es clara la preponderancia de intereses externos a la vida institucional y se reafirma el poder autocrático, centralista y vertical del ejecutivo en la dirección universitaria se consolida la pérdida de autonomía de las instituciones proceso que se venía adelantando en los anteriores gobiernos del Frente Nacional y se fortaleció el estilo de dirección clientelista y tecnócrata al darle al ICFES (Instituto Colombiano de Fomento para la Educación Superior) Instituto descentralizado creado en la década del 60 la función de ejercer la suprema vigilancia y orientación de la educación superior en el país.

El ICFES en su existencia no ha podido fomentar el desarrollo de la Universidad estatal y más bien ha sido un obstáculo para su fortalecimiento académico.

Ha sido muy exigente con las Universidades Públicas especialmente con los de las provincias y muy complaciente con las privadas en este sentido el ICFES ha contribuido a la privatización de la educación superior.

Durante la Administración de Betancourt (1980-1984) se dio un gran impulso a los programas de Educación Abierta y a Distancia. Esta coyuntura facilitó la apertura de un número de programas de esta naturaleza en las universidades especialmente privadas con unas estrategias metodológicas muy deficientes, de baja calidad y donde ha predominado el criterio economicista, ya que la generalidad de los programas ofrecidos se orienta hacia campos donde su implementación genera menores costos. Se favoreció el desarrollo de un muy heterogéneo sector de educación superior destinado a cubrir demandas de acceso con niveles muy discutibles.

Como lo plantea el profesor Queipo Timana "la reforma acrecentó el proceso de parcialización del conocimiento humano, se exigieron una serie de requisitos que obstaculizan el ascenso en la pirámide educacional, lo que va haciendo la Universidad más elitista. Ordenamiento político administrativo de la Universidad que permita una dinámica utilización de recursos y busca disminuir la responsabilidad financiera del estado e implantación de la autofinanciación". (Timana, 1987: 51)

Durante el período se observó un acelerado crecimiento de las instituciones de Educación Superior privadas las cuales cubren el 60.4\% del total de las matrículas, cifra que ubica a Colombia junto con la del Brasil como los países que poseen el mayor número de universidades privadas as de América Latina. 
La educación y la Universidad Pública adolece defectos, pero comparte esa situación con la Universidad privada e incluso, resiste airosa la comparación con el/a en lo que concierne a muchos aspectos. La Universidad privada quizás es más desigual que la pública en lo atinente a calidad o a la orientación. (Mockus, 1992)

No es eje focal de este análisis realizar un diagnóstico de la Universidad Pública, pero consideramos indispensable mencionar algunos aspectos centrales que han incidido en la vida de las Universidades Públicas Colombianas. Las reformas implementadas a partir de los gobiernos del frente nacional al definirlas como institutos públicos descentralizados, no sólo les restringió al máximo un elemento esencial para su trabajo, la autonomía, sino que además las asimiló a las demás dependencias del Estado, lo cual en las Universidades de Provincia las ha convertido en botín politiquero, que las ha ahogado al vaivén de los intereses clientelistas, la asfixia presupuestal en que funcionan ha generado que tanto administradores y docentes se desempeñen en el círculo vicioso de la pobreza, la falta de lineamientos filosóficos claves y por consiguiente de planeación que progresivamente las ha marginado de sus funciones y que hayan perdido imagen en sus comunidades. Por otro lado el ICFES ha utilizado unos indicadores para valorar el prestigio de las universidades, criterios que poco dicen de su calidad como; el número de estudiantes que recibe, la extensión de los terrenos que ocupa, el tamaño de sus edificios o la magnitud de su presupuesto.

Las reformas de la educación superior al producir la simbiosis de la Universidad con el Estado (Decreto 80/80) han llevado a que las Universidades públicas hayan oscilado en el péndulo de la crisis al Estado. Es claro entender que una universidad que se corresponde en su pensamiento con el Estado no tiene nada que hacer. Alejados de la problemática de su región, ignorando sus objetivos, sin poder definir su misión, muchas de nuestras universidades públicas subsisten como un parásito de la sociedad a la cual deben servir, desconociendo su misma razón de ser.

\section{Consideraciones Finales}

La reforma constitucional que se llevó a cabo en 1991 tuvo como un elemento sustancial en la formulación de la nueva carta magna del país, aspectos relacionados con la educación como un derecho ciudadano de primer orden y plantear los fundamentos para su reorganización, financiación, cobertura y mejoramiento cualitativo, como una alternativa para la democratización de la sociedad colombiana. 
Cuando Colombia se encuentra en una etapa de transformación y marcha acelerada en medio de múltiples contradicciones por el modelo neo liberal que ha adoptado o mejor dicho que se ha impuesto.

En este momento coyuntural para el futuro de la educación colombiana, cuando el Congreso ha promulgado las leyes para reformar el Sistema de Educación en Colombia, en el cual se aspira a determinar el rumbo que deberá imprimírseles en todos los niveles con miras a enfrentar un nuevo siglo matizado de extraordinarios cambios en todos los aspectos de la vida.

Estas leyes se inscriben en el marco de las profundas transformaciones que todos los órdenes de la vida se vienen dando en este final de siglo; en los cuales la educación comienza a ser le centro de una revolución tecnológica que se centra en reconocer el valor de ella, como un factor clave en el crecimiento y el desarrollo; lo que ha convertido al conocimiento en el factor de mayor incidencia en los procesos productivos llevando a la creciente desmaterialización de la producción; fenómeno que se refleja con mucha nitidez en los procesos de informatización.

En su contenido las leyes y sus decretos reglamentarios evidencian avances, ambigüedades y aspectos problemáticos.

Entre los avances se reconocen los procesos de autonomía que se le reconocen a las instituciones educativas, la participación de los estamentos básicos de las comunidades educativas en los procesos de planeación, gestión y administración de las instituciones que aunque con restricciones pueden convertirse en un futuro en semillas para consolidar una sociedad democrática. La formalización de los planes de desarrollo educativo que hacen que las políticas educativas deje de ser de los gobiernos de turno y se conviertan en políticas de estado para que puedan ser consolidados.

Las principales ambigüedades se presentan en los aspectos financieros que fortalecen los crecientes ritmos de privatización de las instituciones, los procesos acelerados de descentralización mediante los cuales el poder central transfiere muchas responsabilidades especialmente de carácter financiero y dotación de recursos materiales y didácticos a las administraciones regionales y locales, que en buen número no poseen ni los recursos ni la infraestructura requerida para asumir esas responsabilidades; estas decisiones pueden generar mayores desequilibrio s en la calidad del servicio educativo.

Las políticas de universatización de la educación básica si bien plantean la posibilidad de ampliar el acceso a la educación; no determinan estrategias definidas para enfrentar la problemática de la calidad. Lo que el país requiere es ofrecer a todos los colombianos educación de buena calidad. 
Íntimamente ligado a las consideraciones anteriores está la garantía de una remuneración digna a los educadores en todos los niveles; las disposiciones sobre salarios han privilegiado aspectos relacionados con el control de la inflación como eje directriz para estudiar medidas tendientes a profesionalizar el salario de los docentes, este aspecto ha generado conflictos entre el estado y los educadores tanto de la educación básica como del nivel superior.

Entre los problemas que se observan en las leyes vale la pena destacar (Mejía, 1994: 18) las dificultades que aparecen como fruto del tipo de negociación que se dio para su formulación y de los actores más involucrados directamente en ese proceso de concertación y en otras, aparecen como conceptos y concepciones de un pasado que no quiere desaparecer para dar lugar al nuevo país".

Entre las dificultades que se han señalado vale la pena destacar; la pérdida de un sistema nacional de educación, muchas de las responsabilidades del sector educativo se dispersan en una serie de organismos estatales adscritos a varios ministerios, no se sabe como se van a articular. Primero se promulgó la ley de la educación superior (ley 30 de 1992) y luego la ley general de la Educación (Ley 115 de 1994) entre los cuales se presentan serias incongruencias, se ha planteado articularlos mediante una ley orgánica pero todavía no se conoce su contenido.

Finalmente, la mayoría de los Decretos Reglamentarios han sido expedidos sin una participación de la sociedad, lo que puede llevar a que su aplicación se vuelva regresiva.

En el cual el proyecto educativo entra a formar parte del modelo de globalización e internacionalización de la economía colombiana, en el que se coloca a la educación en el vaivén de las fuerzas del mercado, como cualquier tipo de mercancía que está regulada por las leyes de la competencia del libre comercio.

En este contexto cobra inusitada trascendencia plantearse algunos aspectos fundamentales sobre el quehacer de la educación como instancia de la cultura que interpreta la cosmología social de su época, como depositaria de los valores que se han ido acumulando por la tradición y que en este momento particular se evidencian como uno de los factores determinantes de la crisis de la sociedad colombiana, pero también de los valores y retos de una sociedad dinámica. La Universidad pública como cúspide del sistema educativo especialmente las regionales tienen el imprescindible compromiso con sus comunidades de recuperar su prestigio y sentido de ser como faros que orientan e iluminan el desarrollo de sus contextos. 
Los esfuerzos para articular la Universidad con la educación básica han sido poco relevantes, existe una desarticulación entre ambos sistemas. La calidad y eficiencia de la educación colombiana seguirá siendo limitada mientras no se logré la articulación entre ambos sistemas.

El sistema educativo colombiano debe ser repensado para que pueda ofrecer una educación básica a la mayoría de los habitantes de este país que seguirán siendo sus usuarios por un largo período, una formación para que puedan sobrevivir, desarrollar plenamente sus capacidades, vivir y trabajar con dignidad, participar plenamente en el desarrollo, mejorar la calidad de su vida tomar decisiones fundamentales y continuar aprendiendo tal como lo plantean las conclusiones de la conferencia mundial de Educación realizada en 1990 en JOM - TIEM - Tailandia.

La Universidad Pública tiene que ponerse a tono con las demandas crecientes que se presentan y convertirse en una verdadera institución social que signifique un efectivo instrumento de desarrollo para el país. Debe ser el instrumento de desarrollo y de progreso social, ello debe promover el engrandecimiento de la colectividad que la rodea, lograr la superación de los niveles de vida, determinar su futuro a través de la acción del producto: un individuo formado sobre la base de un conocimiento profundo de su medio, dueño de sólido adiestramiento y con un alto contenido social en sus motivaciones.

Se requiere superar el espíritu de la universidad profesionalizante que ha predominado en Colombia, en donde su misión se ha centrado en especializar profesionales, aislándose de una visión universal del hombre y la cultura y llevándola más bien a un cierto grado de visión restringida del mundo, generado a partir de la dependencia económica, técnica y cultural. No se puede seguir pensando en las Ciencias Humanas y Sociales como especie de complemento del saber científico o técnico, sino como partes integrantes en la construcción y diseño de teorías sobre nuestra historia, nuestra cultura y nuestro futuro. En esta tarea resulta fundamental que los docentes en general y quienes cultivamos las Ciencias Sociales asumamos con decisión el papel trascendental que debe enmarcar el trabajo universitario.

Hay que estimar y potenciar en sus implicaciones qué significa para la democratización política y la participación de las mayorías, preceptos fundamentales de nuestro nuevo orden jurídico, que se agudiza con la segmentación desigual de los consumos, incluida la Educación Superior.

En este aspecto veo uno de los mayores desafíos para la integración, la participación responsable y comprometida de la Universidad colombiana y en especial de los docentes, para contribuir a dilucidar una de las contradicciones más visibles del sistema político de nuestro fin de siglo. 


\section{Bibliografía}

Batallan, Graciela, 1983 Los procesos de interpretación en los talleres de investigación y aprendizaje, Buenos Aires Centro de investigaciones

Cajiao, Francisco, 1987 La formación de maestros y el desarrollo politico del pais, Bogotá: Ponencia presentada al Congreso Pedagógico Nacional

FES Fundación Para la Educación Superior, 1987: La Educación rural en Colombia, Bogotá: FES

Florez O, Rafael y Vladimir Zapata V., 1991: Programa de Estudios Cientifcos de la Educación, Bogotá Propuesta Documento de Trabajo,

Herrera, Martha, 1986 "La educación en la segunda república liberal 19301946” en Revista Colombiana de Educación No 18, Bogotá

Mejia, Marco Raúl, 1994 Ley General de Educación: entre la reglamentación del servicio y la regulación del sistema, Santafé de Bogotá: CINEP

Mockus, Antanas, 1992: Especificidades de la Educación Pública y Reforma Universitaria, Bogotá: Mimeo.

Parra Sandoval, Rodrigo, 1986 La escuela inconclusa, Bogotá Plaza y Janes

Schmelhes, Silvia, 1991: Necesidades básicas de Aprendizaje de los Adultos en América Latina, México: Mimeo

Tedesco, Juan Carlos, 1990: "La Educación y el desarrollo en América Latina” en Documento, Año IV No. 5

Timana, Queipo, 1987: “Una reforma que no reformó la Universidad” en Revista Educación y Cultura No. 12, Bogotá

Timana, Quiepo, 1987 "Una Reforma que no reforma la Universidad en Revista Educación y Cultura No. 12, Bogotá: FECODE 